\title{
HIV-1 mutational pathways under multidrug therapy
}

\author{
Glenn Lawyer ${ }^{* *}$, André Altmann², Alexander Thielen ${ }^{1}$, Maurizio Zazzi ${ }^{3}$, Anders Sönnerborg $^{4}$ and Thomas Lengauer ${ }^{1}$
}

\begin{abstract}
Background: Genotype-derived drug resistance profiles are a valuable asset in HIV-1 therapy decisions. Therapy decisions could be further improved, both in terms of predicting length of current therapy success and in preserving followup therapy options, through better knowledge of mutational pathways- here defined as specific locations on the viral genome which, when mutant, alter the risk that additional specific mutations arise. We limit the search to locations in the reverse transcriptase region of the HIV-1 genome which host resistance mutations to nucleoside (NRTI) and non-nucleoside (NNRTI) reverse transcriptase inhibitors (as listed in the 2008 International AIDS Society report), or which were mutant at therapy start in $5 \%$ or more of the therapies studied.
\end{abstract}

Methods: A Cox proportional hazards model was fit to each location with the hazard of a mutation at that location during therapy proportional to the presence/absence of mutations at the remaining locations at therapy start. A pathway from preexisting to occurring mutation was indicated if the covariate was both selected as important via smoothly clipped absolute deviation (a form of regularized regression) and had a small p-value. The Cox model also allowed controlling for non-genetic parameters and potential nuisance factors such as viral resistance and number of previous therapies. Results were based on 1981 therapies given to 1495 distinct patients drawn from the EuResist database.

Results: The strongest influence on the hazard of developing NRTI resistance was having more than four previous therapies, not any one existing resistance mutation. Known NRTI resistance pathways were shown, and previously speculated inhibition between the thymidine analog pathways was evidenced. Evidence was found for a number of specific pathways between NRTI and NNRTI resistance sites. A number of common mutations were shown to increase the hazard of developing both NRTI and NNRTI resistance. Viral resistance to the therapy compounds did not materially effect the hazard of mutation in our model.

Conclusions: The accuracy of therapy outcome prediction tools may be increased by including the number of previous treatments, and by considering locations in the HIV genome which increase the hazard of developing resistance mutations.

\section{Background}

Antiretroviral treatment has turned infection with the Human Immunodeficiency Virus (HIV-1) into a manageable disease. Yet eventually the HIV variants circulating in the patient develop resistance to the applied drugs. In many cases, it is known which mutations give resistance to which drugs, allowing accurate prediction of therapy efficacy based on HIV genotyping [1], with generally good results $[2,3]$. Better understanding of

\footnotetext{
* Correspondence: lawyer@mpi-inf.mpg.de

'Department of Computational Biology, Max Planck Institute for Informatics, Saarbrücken, Germany

Full list of author information is available at the end of the article
}

which pre-existing mutations effect the development of resistance would further improve treatment, informing both choice of compounds for the current therapy and long-term strategies to maintain treatment options when the current therapy fails.

Reverse transcriptase inhibitors (RTIs) are the longest used and arguably the most important class of antiretrovirals. These compounds inhibit the reverse transcription of single-stranded viral RNA into double-stranded viral DNA suitable for incorporation into the host DNA. They are classified as either nucleoside (NRTIs), which incorporate into and terminate transcription of the viral DNA, or non-nucleoside (NNRTIs), which change the

\section{Biomed Central}


conformation of the RT polymerase into a non-functional state. RTIs are expected to remain a critical therapy component even as new classes of drugs, such as entry and integrase inhibitors, are added to the anti-HIV arsenal [4].

Accordingly, a great deal of work has investigated development of RTI resistance. Many RTI resistance mutations are known to occur in clusters [5]. Two of the most studied NRTI clusters are the thymidine analog resistance mutations, TAM-1 (41L, 210W, 215Y) and TAM-2 (67N, 70R, 215F, 219E/Q), [6]. which show evidence of appearing in ordered sequence $[6,7]$. Less evidence supports pathways to NNRTI resistance, which can arise from a single mutation [8] with little impact on viral fitness [9-11]. Data from clinical trials of efavirenz (an NNRTI), however, suggested that mutation at location 103 preceded mutation at locations 100, 101, 108 , and $225[12,13]$.

Standard of care generally dictates two NRTIs supplemented with additional compounds which may include an NNRTI. Understanding of the development of resistance under such multidrug regimes is far from complete $[14,15]$. It has been shown that subjects with NNRTI resistance were at greater hazard of developing NRTI resistance, and vice versa [16], but not which specific factors explained this. Several sources have indicated interactions and other forms of crossplay between NRTI and NNRTI resistance mutations, but have not demonstrated clear pathways $[4,17]$.

Many of the mutations which commonly occur during therapy do not have a known, direct connection with drug resistance. In the data studied here, 45 different locations frequently harbored mutations at therapy start; 32 of these are not on the International AIDS Society list of RTI resistance mutations [18]. Patterns within these other mutations may underly the just commented on interplay, lending high interest to pathways leading from commonly mutant locations to known resistance sites.

We place the question of identifying mutational pathways in a survival analysis framework. A mutational pathway from (genetic) location $a$ to $b$ is signalled if mutation at location $a$ alters the hazard of mutation at location $b$. Survival analysis extends the specificity of investigations based on co-occurrence of mutations (i.e. $[4,7,17])$ by indicating both excitatory and inhibitory influences, incorporating temporal dynamics, and making full use of the data despite the abundant censoring. The framework further allows for control of nuisance parameters which are inherent in clinical data. In the current case, the most important of these is having a high number of previous therapies. While other techniques from survival analysis have been previously applied to RTI resistance $[11,13,16]$, and protease inhibitor resistance [19], this is, to our knowledge, the first use of such methods to directly addresses the question of specific mutational pathways.

We tested for pathways between known resistance sites, and also for pathways between commonly mutant locations. Pathways were signalled by a two stage filtering process. For a given mutational site, we first applied smoothly clipped absolute deviation (SCAD) [20], a form of regularized regression, to identify a subset of pre-existing mutations which showed evidence of influencing the hazard of mutation at that site. These were further screened via standard significance testing to identify those with strong evidence for effect. The model also tested for effects associated with the clinical variables. Longitudinal data were available from the EuResist database [21]. EuResist maintains, to our knowledge, the largest HIV resistance database available for public research.

\section{Methods}

\section{Subject material}

Subject material for this study was drawn from the EuResist database [21]. The EuResist project integrates viral genotypes, therapy, and patient data collected by hospitals throughout Europe, notably from Italy, Germany, Sweden, Belgium, Spain, Portugal, and Luxembourg. Our study was based on therapy records which contain genotypes recorded both at therapy start and before therapy end, and which included an RTI. While the EuResist database was not designed with this desiderata, the 2010-01-26 release contains 1981 RTIbased therapies for which HIV genotype was recorded up to three months prior to therapy start, and a second genotype recorded before the end of said therapy. These therapies represent 1495 unique subjects. Two hundred and seven subjects appear twice, and 102 subjects appear multiple times. Table 1 lists the ten most frequently prescribed combinations of RTIs; the full list is given in the supplement [see Additional file 1, Table S1].

The outcome measure of the current study was the presence of mutation at a second genotype taken before therapy end. The distribution of the time delay between the first and second genotyping was approximately equally across the different risk groups [see Additional File 1, Figure S1]. The use of a second genotyping is subtly, but crucially, distinct from using time of therapy failure as an outcome measure. Twenty percent of the therapies were ongoing at the time the second genotype was recorded. Further, the EuResist database defines a therapy based on the compounds given. Therapies are considered to end when any compound in the therapy is added or removed, regardless of virological suppression. Often the cause is therapy change is not recorded. Table 
Table 1 Therapy profiles

\begin{tabular}{llll}
\hline \multicolumn{4}{c}{ Therapy profiles } \\
\hline Compounds & $\mathbf{N}$ & Duration & \# Previous \\
\hline 3TC AZT & 259 & $549(21,3515)$ & $3(0,28)$ \\
\hline d4T DDI & 149 & $553(40,3291)$ & $5.2(0,19)$ \\
\hline TDF FTC & 123 & $284(28,1122)$ & $5.9(0,25)$ \\
\hline 3TC d4T & 115 & $646(19,3508)$ & $4.5(0,18)$ \\
\hline 3TC TDF & 96 & $311(27,1360)$ & $7.4(0,20)$ \\
\hline 3TC DDI & 61 & $590(43,2268)$ & $8.6(1,37)$ \\
\hline 3TC ABC & 52 & $397(40,1140)$ & $6.5(0,18)$ \\
\hline AZT & 50 & $379(1,1939)$ & $2(0,12)$ \\
\hline 3TC ABC AZT & 50 & $437(28,1423)$ & $4.6(0,19)$ \\
\hline AZT DDI & 49 & $413(57,1408)$ & $5.1(0,17)$ \\
\hline
\end{tabular}

The data in the current study contains 119 unique combinations of reverse transcriptease inhibitors. The 10 most common combinations are listed here, along with the number of subjects receiving them, mean (range) duration in days, and mean (range) number of previous therapies when administered. Note that these therapies may also have included protease inhibitors. The full table is given in the supplement.

2 , therapy stop causes, indicates that $51 \%$ of the current therapies do not have a recorded stop cause. Only $19 \%$ of the second genotypings are unequivocally associated with therapy failure.

All genotypes are population sequences reflecting the consensus HIV-1 genotype at the time of measurement. Subject demographics (shown in Table 3) are heterogeneous, and all major risk groups are well represented. The median number of previous therapies is 4 , and ranges from no previous treatments (249 subjects) to 37 previous treatments (1 subject). The reason for including therapy naive subjects is that a pathway is defined by an increase in risk of developing a resistance mutation based on pre-existing mutations. Including therapy naive subjects in the model gives a better estimate of the baseline hazard estimate.

Table 2 Therapy stop causes

\begin{tabular}{lll}
\hline & Therapy stop causes \\
\hline Cause & count & percent \\
\hline Unknown & 1026 & 0.52 \\
\hline Ongoing & 398 & 0.20 \\
\hline Failure & 372 & 0.19 \\
\hline Side effects & 67 & 0.03 \\
\hline Change of therapy & 57 & 0.03 \\
\hline Adherence & 38 & 0.02 \\
\hline Supervised Interruption & 23 & 0.01 \\
\hline TOTAL & 1981 & \\
\hline
\end{tabular}

EuResist defines a therapy based strictly on the compounds included. Any change in compounds is recored as a therapy change. The reason for therapy change was recorded for $75 \%$ of the records included here, and are listed in the database under the following categories.
Table 3 Patient profile

\begin{tabular}{lll}
\hline \multicolumn{3}{c}{ Patient demographics } \\
\hline Age 1st genotyping & 39.7 years & \pm 9.3 \\
\hline Gender (M/F) & $1054 \mathrm{M}$ & $433 \mathrm{~F}$ \\
\hline Num prev therps & 4 (median) & $0-32$ (range) \\
\hline Days between genotypings & 485 (mean) & 639 (variance) \\
\hline Risk group: & & \\
\hline Heterosexual & 450 & $30 \%$ \\
\hline Homo/bisexual & 367 & $25 \%$ \\
\hline IVDA & 372 & $25 \%$ \\
\hline Vertical transmission & 33 & $2 \%$ \\
\hline Blood products & 25 & $2 \%$ \\
\hline Other/unknown & 245 & $16 \%$
\end{tabular}

Background data on the subjects included in the study. When a subject has contributed multiple therapy records to the study, the demographic information is taken from the first therapy included.

\section{Binarization and locations (codons) considered}

This study investigated locations (or codons) known to harbor RTI resistance mutations and locations which were commonly mutant in the EuResist data. Known resistance sites were drawn from 2008 International AIDS Society list of mutations associated with antiretroviral drug resistance [18]. This included the following locations: NRTI: 41, 62, 65, 67, 69, 70, 74, 75, 77, 115, 116, 151, 184, 210, 215, 219 and NNRTI: 100, 101, 103, $106,108,181,188,190,225$. Commonly mutant locations were defined as those which were mutant at the start of $5 \%$ or more of the therapies studied here, namely: $20,35,39,41,43,44,49,60,67,68,69,70,74$, $83,98,101,103,118,122,123,135,142,162,166,169$, 173, 174, 177, 178, 179, 181, 184, 190, 196, 200, 202, 203, 207, 208, 210, 211, 214, 215, 219, and 228. Figure 1 (known resistance) and Figure 2 (commonly mutant) show the frequency of mutation at these locations, both at therapy start and at the second genotyping, for each of the major patient risk groups.

During this investigation only the location was specified and not the amino acid substitution. The assumption is that mutations detectable by population sequencing would be heavily influenced by treatment history. Binarization offered several additional advantages. It simplifies ambiguities arising from the genotyping method. Further, the study included commonly mutant locations not necessarily listed as important to resistance and thus with little literature support to decide which substitutions were relevant. Binarization allowed these locations to be treated identically to the known resistance mutations.

A location was considered to have a preexisting mutation if its genotype at therapy start did not completely agree with the wild type. For example, a location with wild-type " $M$ " which showed the mixture "MV" at 

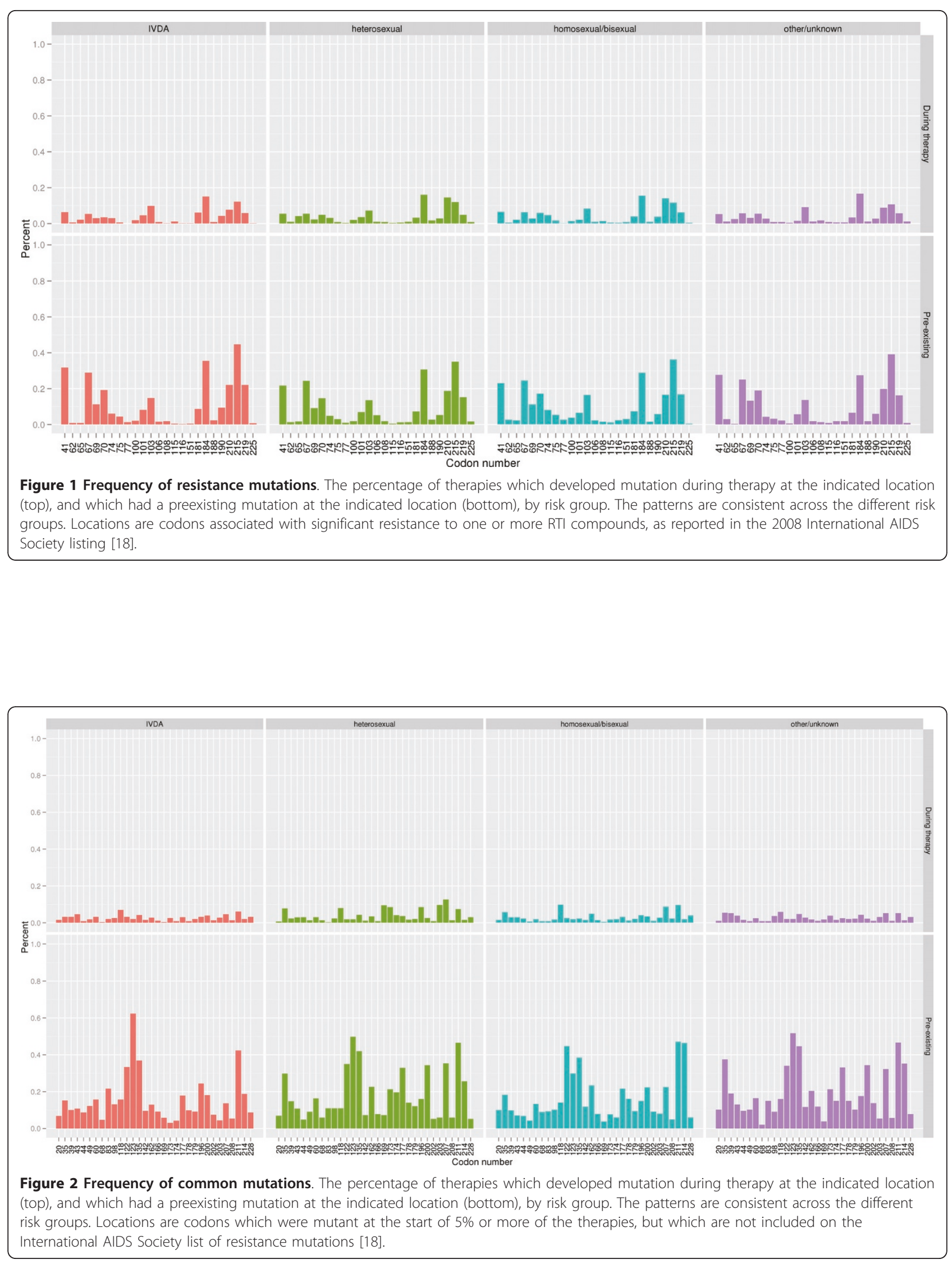
therapy start would be coded as a mutation. A mutation was considered to have occurred during therapy if the observed sequence did not contain an amino acid observed in the preexisting sequence and was not wild type. A preexisting "ML" which changed to "L" during the course of therapy would not be considered a mutation, while a change to " $R$ " would be.

\section{Identifying mutational pathways-basic framework}

Given a location of interest $c$, the goal was to identify other locations where preexisting mutations significantly altered the hazard of developing a mutation at $c$. The influence could be either inhibitory or excitatory. The question was formulated in terms of the Cox proportional hazards model using regressors to signal the presence/absence of preexisting mutations and to control for nuisance parameters. Formally,

$$
h c_{i}(t)=h c_{0}(t) \exp \left(p_{1, i}+\ldots+p_{j, i}+n u i s_{a, i}\right),
$$

where $h c_{i}(t)$ is the hazard that location $c$ becomes mutant in subject $i$ during therapy and $h c_{0}(t)$ is the baseline hazard of mutation at $c$. The Cox model does not require specification of $h c_{0}(t)$, which is integrated out during the model fitting. A preexisting mutation at location $j^{\text {th }}$ in subject $i$ is coded by $p_{1, i} \ldots p_{j, i}$. Having the median or fewer previous therapies was coded by nuis $a_{a, i}$. Other potential nuisance factors, such as the total number of mutations, viral resistance to the therapy compounds, and treatment start year were deemed noninformative in preliminary investigations. An event was signalled when location $c$ was mutant in subject $i$ at the second genotype but not the first. The time to event was the number of days between the two genotypes. While the direction of effect is likely to be correct, this simplifying assumption regarding time to event implies that estimates of magnitude should be regarded with caution.

Within this framework, identification of pathways reduces to a variable selection problem; selecting those regressors with strong evidence of effect on the hazard of developing the target mutation. We first filtered the list of potential regressors using smoothly clipped absolute deviation (SCAD) [20]. SCAD is a form of regularized regression, similar to the LASSO, but with the added benefit that the regularization parameter scales with the magnitude of the regression coefficient. The regressors included in the best SCAD model were then tested for significance using the Wald estimate, and those with $p<0.01$ were deemed to have sufficient evidence to suggest a pathway.

While each individual model could only detect onestep pathways (i.e. pre-existing mutation at locations $p_{3}$ and $p_{7}$ increased the hazard of mutation at $c$ ), fitting the model to each of the candidate regressors in turn produces an adjacency matrix which can be viewed as a directed graph allowing multi-step pathways. We first searched for pathways among known resistance sites by considering the combined list of NRTI and NNRTI resistance locations. We then searched for pathways in our list of commonly mutant locations.

Statistics and figures were created in the R software environment, version 2.7.1 [22]. SCAD was implemented using the R package SIS [23]. Cox model fitting and the Wald estimate were performed using the $\mathrm{R}$ package survival [24]. Visualization was aided by the packages ggplot2 [25] and igraph [26].

\section{Identifying mutational pathways - specific models}

The basic model identifies pre-existing mutation with strong evidence for effect on the hazard of developing mutation at one specific target location $c$. Given this location, therapy data is restricted to those at risk of developing mutation at $c$, that is, those whose HIV genotype did not exhibit mutation at $c$ at the start of treatment. When $c$ was an NNRTI resistance location, therapies were further restricted to those receiving an NNRTI. Note that all of the therapies under consideration included NRTIs. Candidate regressors were also dependent on $c$. Obviously $c$ itself could not be a candidate. Further, some locations never exhibited a preexisting mutation in at-risk therapies, and were dropped to prevent convergence issues. Finally, if data is not available on a specific pre-existing mutation for more than 10 of the at-risk therapies, it was dropped from the model.

\section{Results and Discussion}

The single factor which most consistently influences the hazard of mutation at locations with known involvement in NRTI resistance is the number of previous therapies. The median number of previous therapies in the data studied here is 4 . Therapies for patients with 4 or less previous therapies are associated with less than half the risk of developing RT resistance. The effect is observed at 9 out of 16 locations associated with NRTI resistance: codons $65,67,69,70,74,115,210,215$, and 219 . The median hazard ratio is 0.37 (ranging from 0.15 to 0.52 ), with the lowest $95 \%$ confidence bound at 0.06 and the highest at 0.81 . This effect is not due to the presence of more known resistance mutations in patients with a large number of previous therapies, as known resistance mutations were regressed out by the model. In addition, further testing indicated that genotypically estimated viral resistance has negligible effect in our models. The finding could, however, represent the accumulation of mutations in regions about which little is known because they are rarely sequenced. For example, the first 
investigation of mutations in the connection and ribonuclease $\mathrm{H}$ domains of RT has shown that such mutations strongly influence AZT resistance in combination with the TAM pathways [27].

The study clearly substantiates the well established TAM pathways. The TAM-1 pathway is demonstrated by the observation that mutation at location 215 increases the hazard of mutation at location 41. The TAM-2 pathway is supported by finding that mutation at location 67 increases the hazard of mutation at locations 70 and 219, and that mutation at 70 increases the hazard at 219. All of these pathways involve an estimated three-fold increase in hazard. (Estimated hazard ratios for all indicated mutational pathways which end at known resistance sites are given in Tables 4 and 5 . Hazard ratios for pathways which end at commonly mutant locations are listed in the supplement [see Additional file 1, Table S2]. Our results concur with biological assays suggesting that 215 precedes 41 in development of the TAM-1 pathway, [6], and that $67 \mathrm{~N}$ and 70R are the first mutations to appear in the TAM-2 pathway [27]. The pathway order as determined by the current model largely agrees with that determined by

Table 4 Hazard Ratios for pathways between known resistance locations

\begin{tabular}{lll}
\hline \multicolumn{3}{c}{ Hazard ratios between known resistance locations } \\
\hline Pathway & Hazard & Confidence Bounds \\
\hline $41 \rightarrow 108$ & 7.54 & $(2.2,25.8)$ \\
\hline $67 \rightarrow 70$ & 3.52 & $(2.08,5.98)$ \\
\hline $67 \rightarrow 190$ & 3.19 & $(1.41,7.21)$ \\
\hline $67 \rightarrow 219$ & 2.97 & $(1.72,5.14)$ \\
\hline $70 \rightarrow 210^{*}$ & 0.33 & $(0.15,0.75)$ \\
\hline $70 \rightarrow 219$ & 2.68 & $(1.6,4.47)$ \\
\hline $74 \rightarrow 100$ & 12.92 & $(3.48,47.95)$ \\
\hline $77 \rightarrow 103$ & 7.76 & $(2.6,23.16)$ \\
\hline $115 \rightarrow 106$ & 17.51 & $(1.26,243.88)$ \\
\hline $116 \rightarrow 62$ & 27.81 & $(8.73,88.58)$ \\
\hline $151 \rightarrow 116$ & 237.67 & $(34.71,1627.63)$ \\
\hline $181 \rightarrow 65$ & 3.28 & $(1.22,8.81)$ \\
\hline $184 \rightarrow 181^{\dagger}$ & 1.77 & $(1.07,2.92)$ \\
\hline $184 \rightarrow 210$ & 0.27 & $(0.17,0.42)$ \\
\hline $190 \rightarrow 184$ & 1.84 & $(1.15,2.95)$ \\
\hline $210 \rightarrow 70$ & 0.09 & $(0.03,0.23)$ \\
\hline $215 \rightarrow 41$ & 3.23 & $(2.15,4.85)$ \\
\hline $215 \rightarrow 65$ & 0.06 & $(0.01,0.28)$ \\
\hline
\end{tabular}

Estimated hazard ratios and 95\% confidence bounds for all pathways between known resistance locations with $p<0.01$. Two additional pathways, which were remarked on in the text, are also included. Estimation was done using the survival [24] package of the R software environment [22].

${ }^{*} p=0.011{ }^{\dagger} p=0.019$
Table 5 Hazard Ratios for pathways from commonly mutant locations to known resistance locations

\begin{tabular}{lll}
\hline \multicolumn{3}{c}{ Hazard ratios; common to resistance locations } \\
\hline Pathway & Hazard & Confidence Bounds \\
\hline $43 \rightarrow 103$ & 1.92 & $(0.85,4.35)$ \\
\hline $49 \rightarrow 103$ & 0.46 & $(0.20,1.07)$ \\
\hline $67 \rightarrow 70$ & 4.66 & $(2.17,9.99)$ \\
\hline $67 \rightarrow 190$ & 3.31 & $(1.70,6.42)$ \\
\hline $68 \rightarrow 184$ & 1.93 & $(1.21,3.08)$ \\
\hline $70 \rightarrow 103$ & 1.01 & $(0.49,2.08)$ \\
\hline $70 \rightarrow 181$ & 0.37 & $(0.15,0.92)$ \\
\hline $70 \rightarrow 219$ & 2.76 & $(1.61,4.74)$ \\
\hline $74 \rightarrow 184$ & 1.84 & $(1.07,3.17)$ \\
\hline $118 \rightarrow 219$ & 1.62 & $(0.83,3.14)$ \\
\hline $135 \rightarrow 210$ & 0.38 & $(0.25,0.59)$ \\
\hline $142 \rightarrow 67$ & 1.89 & $(1.13,3.16)$ \\
\hline $162 \rightarrow 70$ & 1.89 & $(1.08,3.31)$ \\
\hline $179 \rightarrow 103$ & 1.98 & $(0.97,4.02)$ \\
\hline $184 \rightarrow 181$ & 1.92 & $(1.13,3.27)$ \\
\hline $184 \rightarrow 190$ & 0.73 & $(0.39,1.39)$ \\
\hline $196 \rightarrow 103$ & 1.87 & $(1.14,3.05)$ \\
\hline $196 \rightarrow 190$ & 1.90 & $(0.92,3.92)$ \\
\hline $196 \rightarrow 210$ & 0.47 & $(0.24,0.93)$ \\
\hline $200 \rightarrow 190$ & 1.76 & $(0.92,3.35)$ \\
\hline $210 \rightarrow 70$ & 0.12 & $(0.04,0.34)$ \\
\hline $211 \rightarrow 210$ & 0.46 & $(0.31,0.68)$ \\
\hline $215 \rightarrow 41$ & 2.69 & $(1.44,5.03)$ \\
\hline
\end{tabular}

Estimated hazard ratios and 95\% confidence bounds for pathways leading from commonly mutant locations to locations with known association to RT resistance. Slight discrepancies in estimated hazard ratios between this table and Table 4 are due to a different choice of regressors used to fit the model. Estimation was done using the survival [24] package of the R software environment [22].

the mutagenetic tree model both as applied to the current data and as in the original publication [7].

Inhibition of TAM-2 by TAM-1 is also observed. Mutation at location 210 is associated with a tenfold reduction in the risk of mutation at 70 . A similar effect is witnessed in the reverse direction, though the reduction is only threefold, and the significance level is just above threshold $(p=0.011)$. The to date most thorough report on the TAM pathways [6] presented speculative evidence that TAM-1 inhibits TAM-2. Independent support for this inhibition was presented by Sing et. al [28]. Other studies, however, have reported that some patients develop mutations in both TAM clusters, or switching from TAM-1 to TAM-2 [29].

No pathways are seen between NNRTI resistance locations. This was not unexpected, as in vitro investigations suggest that resistance to most NNRTIs can result 
from a single mutation [8]. This evidence is corroborated by a Bayesian analysis of combinatorial mutation patterns which indicates that interactions among mutations granting nevirapine (an NNRTI) resistance were very weak [30]. Pathways have been suggested, however, in data from a clinical trial of efavirenz $[12,13]$.

The method also indicated several cross-class resistance pathways. Specific pathways from NRTI to NNRTI resistance included the following, all of which showed multi-fold increase in hazard: $41 \rightarrow 108,67 \rightarrow$ $190,74 \rightarrow 100$, and $77 \rightarrow 103$. Previous work has observed that mutation at 74 is associated with increased frequency of NNRTI failure [4]; mutation at location 100 grants resistance to most NNRTIs [18]. Some evidence also suggests that the L74V mutation compensates loss of viral fitness incurred by the double NNRTI resistance mutations L100I + K103N [31].

A pathway was suggested from 184 to 181 , though the associated p-value (0.019) is above our threshold. This finding is disconcerting, as mutation at 184 is one of the most common routes to NRTI resistance, and mutation at 181 grants resistance to all NNRTIs [18]. Specific NNRTI to NRTI resistance pathways are $181 \rightarrow 65$ and $190 \rightarrow 184$. Bayesian networks have suggested robust dependencies between NRTI mutations at 65, 74, 75, and 184 and NNRTI mutations at 100, 181, 190, and 230 [17], though the pathways suggested by the current work are not explicitly implicated in [17]. The adjacency matrix describing the pathways indicated in the current study is given in Figure 3, a network representation is given in Figure 4.

Survival analysis has shown that having any (N)NRTI resistance mutation increases the hazard of developing a mutation in the other class, [16]. We note that Healy et al.'s findings of general dependence showed stronger effect sizes than ours. This slight divergence could be dependent on the selection of subjects. Most of Healy et al.'s subjects had few previous treatments, while the EuResist subjects had failed a median of four previous therapies. As the EuResist subjects had a number of accumulated mutations, the risk profile of mutations which could arise in these subjects is likely to differ

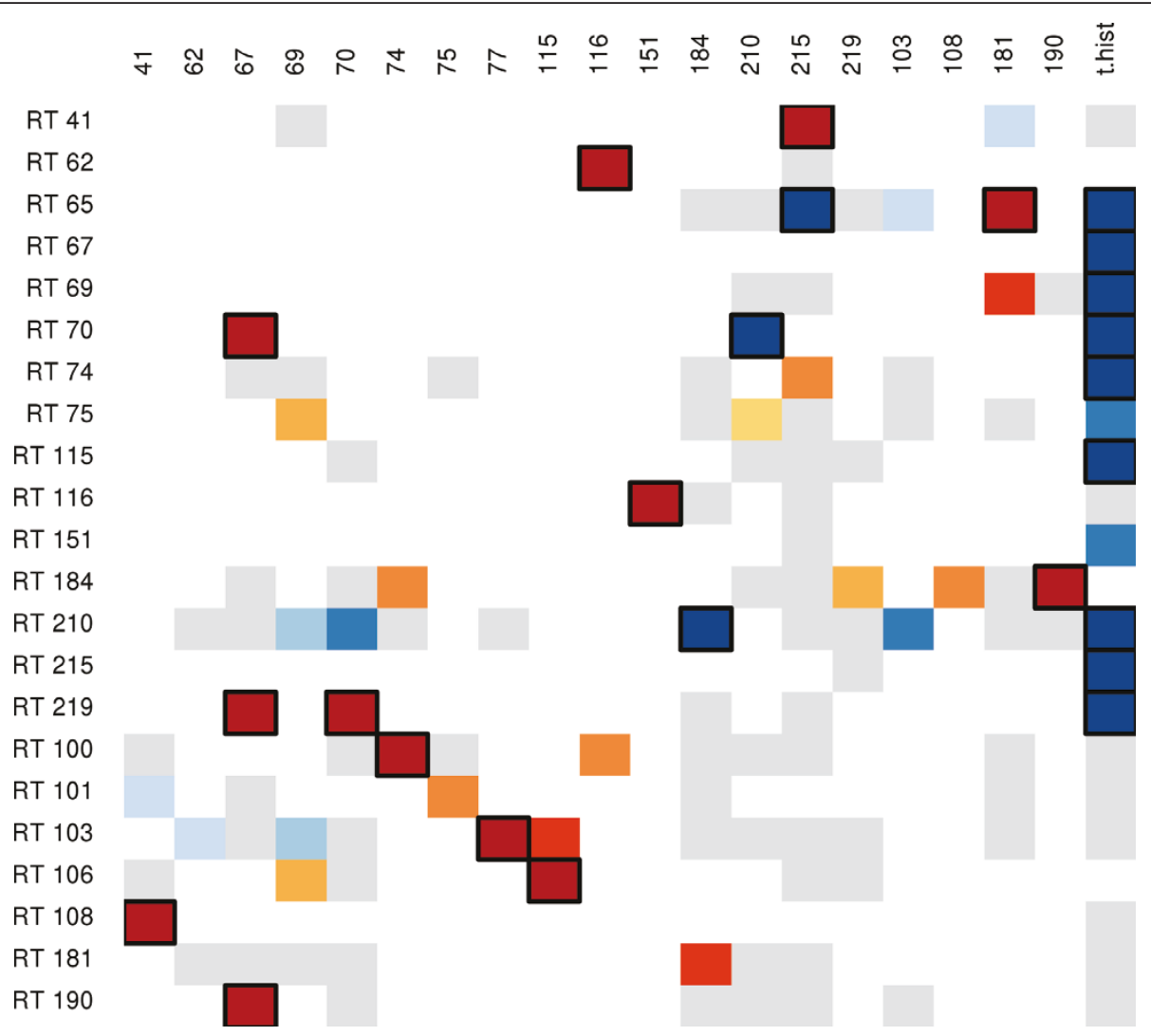

Figure 3 Adjacency matrix of pathways within RTI resistance locations. Columns are pre-existing mutations, rows are the outcome mutation. The matrix has been reduced to only include rows or columns with at least one detected effect. Colored cells are those which were suggested by SCAD. Grey cells had $p>0.05$; the remaining cells are colored based on p-value, with darker colors indicating lower p-values. Red colors indicate a pre-existing mutation was associated with an increase in hazard, blue indicates a reduction. A box has been drawn around cells with $p<0.01$. 


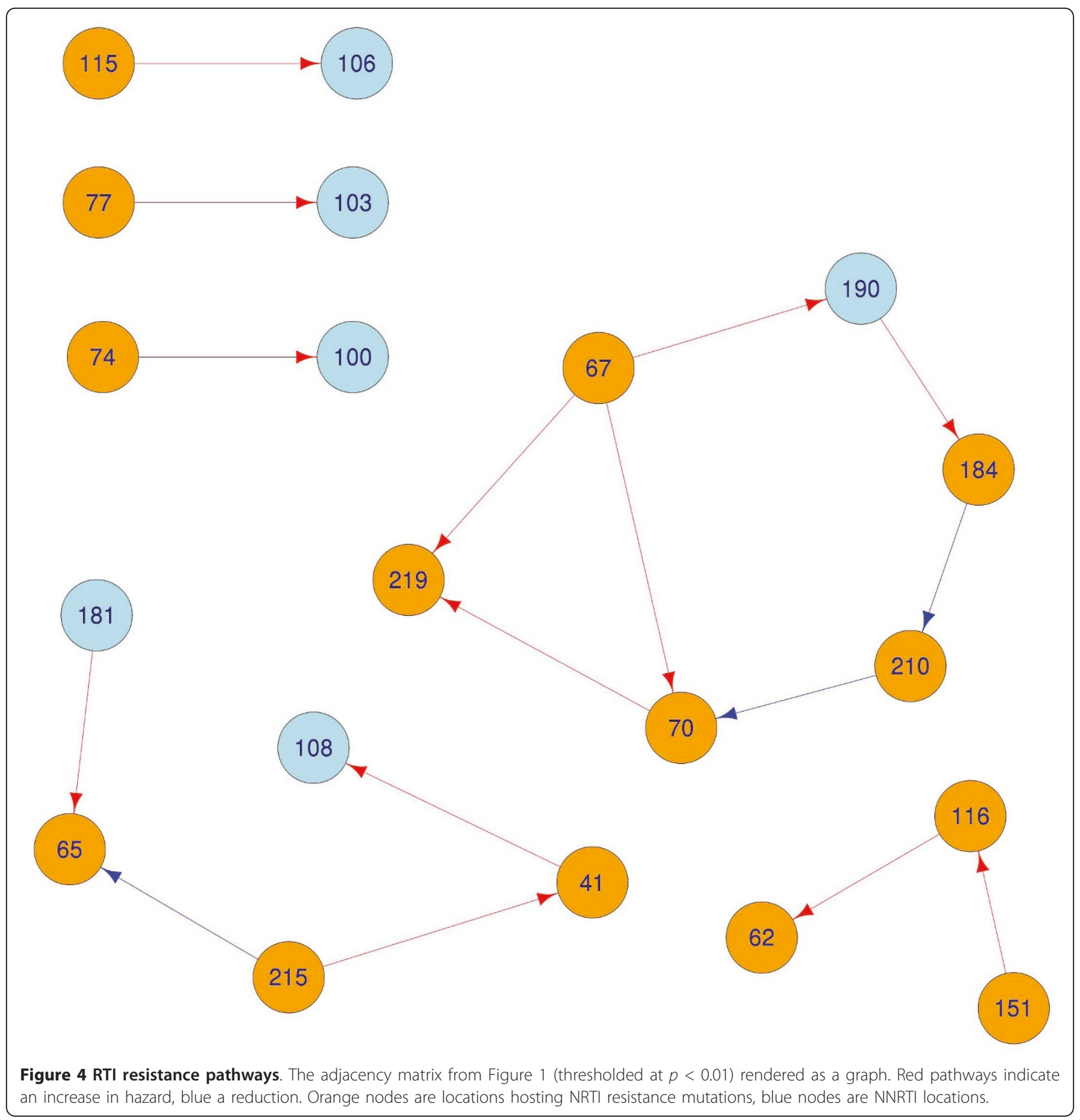

substantially from Healy et al.'s subjects. The evolutionary dynamics might also differ between the two groups. The accumulated mutations in the HIV variants circulating in patients with a long treatment history are likely to have reduced the virus's replicative capacity [32]. Differences could also be specific to viral subtype. We note that neither Healy et. al. nor the report of the clinical trial which supplied their data provide subtype information. Finally, Healy et al.'s data came from a prospective study, whereas the EuResist data is retrospective.
Commonly mutant locations were defined as those locations which hosted a mutation at the start of at least $5 \%$ of the therapies analyzed in this study. Of the 45 locations which met this criteria, 13 are known to harbor resistance mutations. Fifteen edges lead out from known resistance sites. Five of these connect to locations not associated with resistance. The patterns observed above in the known resistance mutations are mostly preserved in this list. This should not be regarded as an independent observation. Though the candidate regressors are different, the data is 
the same. The adjacency matrix describing pathways in commonly mutant locations is given in Figure 5, a network representation is given in Figure 6.

Pathways which lead to known resistance sites could prove informative in predicting the development of (further) resistance. A number of pathways to NRTI resistance sites begin from locations not listed as providing RTI resistance, though the estimated increase in hazard is in general lower than that observed between known resistance mutations (see Table 5). Their influence suggests that therapy outcome prediction engines could be improved by incorporation of the following pathways: $142 \rightarrow 67,214 \rightarrow 69$, and $162 \rightarrow 70$. It was also observed that $68 \rightarrow 184$, and that mutation at either 177 or 181 increases the hazard of 68 . This final observation suggests an indirect $181 \rightarrow 68 \rightarrow 184$ pathway from NNRTI to NRTI resistance. An inhibitory pathway was also identified, with mutation at 135 reducing the hazard of mutation at 210 by a factor of 0.38 (95\% confidence bounds of $0.25,0.59$ ).

Several pre-existing mutations are associated with increased hazard for mutation granting NNRTI resistance, concurring with previous research suggesting that pathways to NNRTI resistance may start from

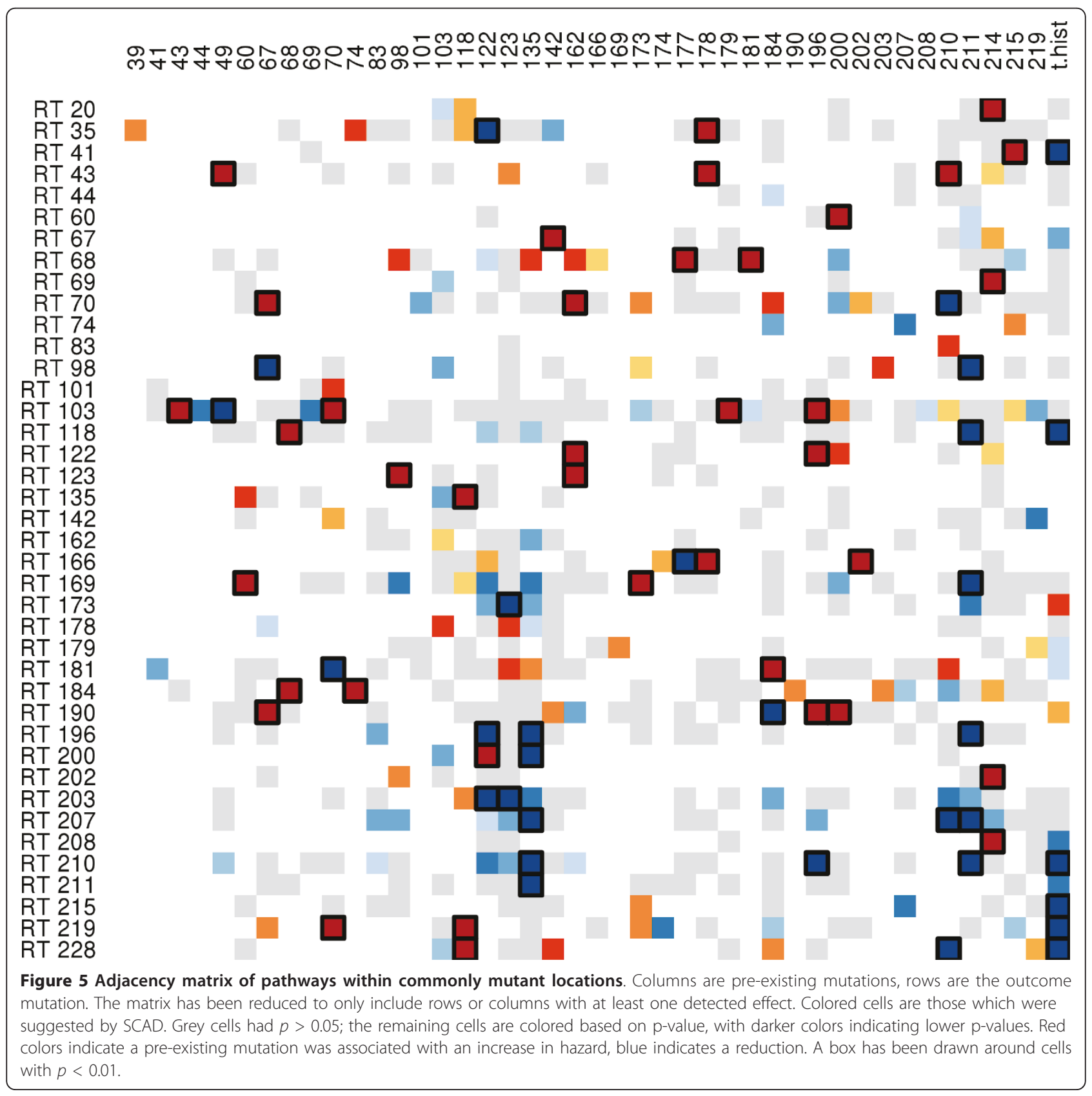




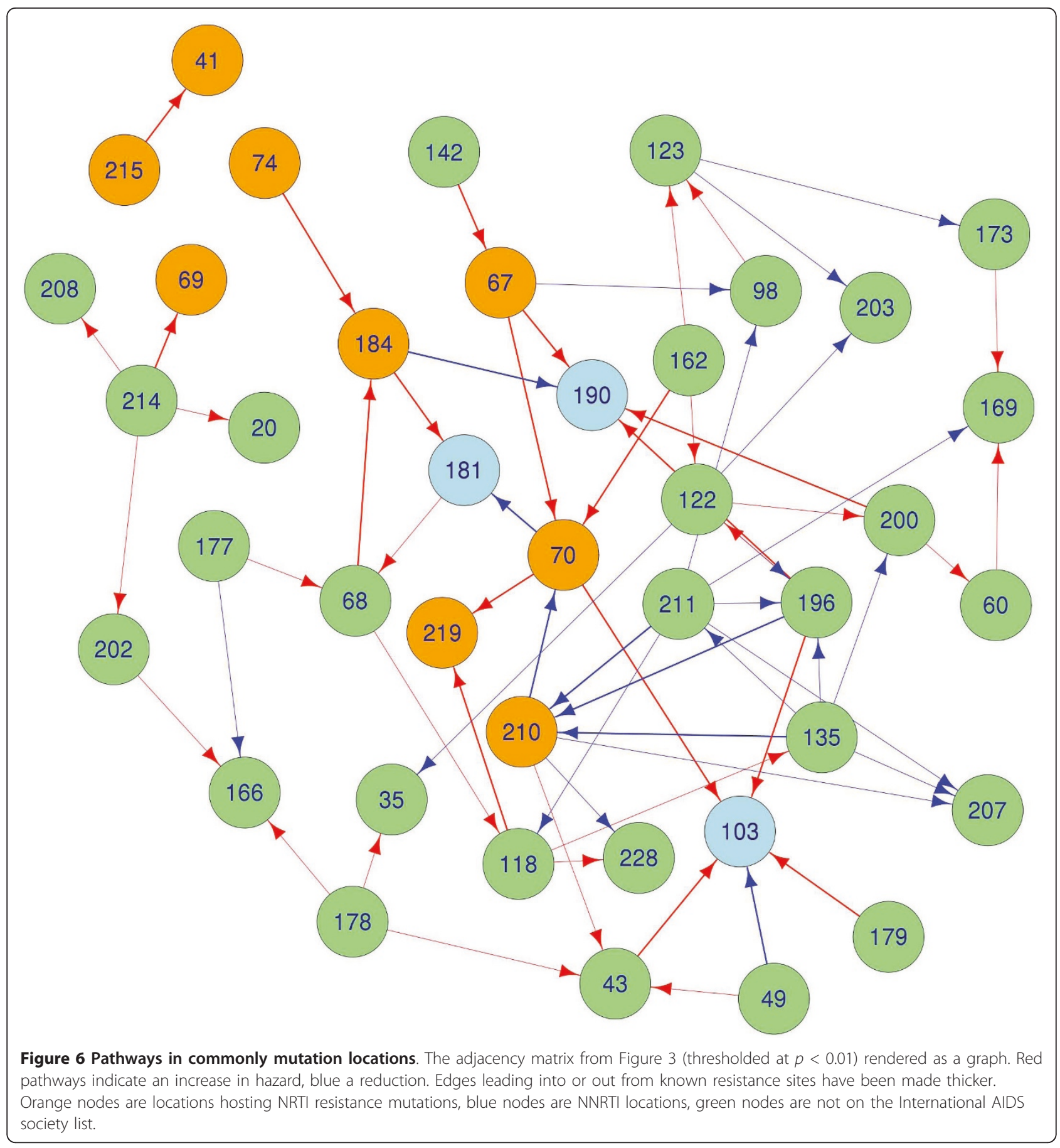

previously unsuspected mutations [4]. Notably, mutation at any of 43,179,or 196 increases the hazard of mutation at 103 .

196 or 200 increase the hazard at 190 . Mutation at location 103 or 190 grants strong resistance to both EFV and NVP. This again suggests that consideration might be given to mutations at locations 43, 179, 196, or 200 before prescribing these NNRTIs. Location 43 could play a part in NRTI to NNRTI resistance, since $210 \rightarrow 43 \rightarrow 103$. The $184 \rightarrow 181$ pathway suggested in the known resistance sites was again observed, and now with sufficient evidence to pass our threshold.

Several mutations seemed to decrease the hazard of NNRTI resistance. Notably, mutation at location 70 (part of the TAM-1 complex granting NRTI resistance) strongly inhibits mutation at location 181. Location 
184 was observed to slightly inhibit mutation at location 190.

This work is, as far as we know, the first to fully employ the Cox model to identify specific mutational pathways. The proportional hazards approach is more sophisticated than methods which do not include time to event or censored events and which cannot control for nuisance parameters. Modeling was simplified, however, by assuming right censored data whereas interval censoring better describes the process generating the data. This simplifying assumption was required as we had no good indication of what an appropriate interval would be. It was justified in that the principle benefit of interval censoring is a more accurate estimate of effect size, not effect significance. Effect size did not play a role in our estimation of pathways.

Some evidence suggests that viral subtype also effects hazard of mutation. Several studies have observed a relationship between resistance mutations and viral subtype, as reviewed in [33] and [34]. A difficulty with such studies, however, is that the data on non-B subtypes tends to come from resource-limited regions where treatment regimes do not always meet European standards of best clinical care. This situation is likely to change as the demographics of the disease change; the UK Health Protection Agency now reports that the majority of UK infections are non-B [35]. Also, molecular dynamics suggest that different patterns of DNA synthesis in subtype $C$ variants compared to subtype $B$ increase the hazard of developing the K65R mutation [36]. Since subtype prevalence varies greatly by patient risk category, it is possible that analysis of subjects in the heterosexual risk group might demonstrate different pathways, or the same pathways with different hazard ratios, than observed in the full data. On the other hand, binarization of the data may have reduced this effect. The V106M resistance mutation may be preferred over V106A in subtype C [37]; the current study gives both of these events the same encoding. Preliminary analysis found subtype to be non-informative to the current model as applied to the EuResist data.

Data for the study came from a large observational database. This can lead to several forms of bias in the data. While we believe these effects to be small, they should be noted. The first bias deals with model assumptions. Our data was censored at the time of the second genotyping. This event is not necessarily independent from our outcome measure, the hazard of mutation. Dependence between the two, however, is difficult to ascertain. The bias would be strongest when the genotyping was conducted due to virological failure of the therapy, a factor which would increase the hazard of additional mutation. On the other hand, virological failure could be caused by the development of resistance mutations, which are present in the model and whose effect is therefore already included in the calculations.
The remaining biases are due to the clinical practices which underly the data gathering. For some, and perhaps most, of the therapies, genotyping was used to select treatment. Neither drug choices nor drug combinations were random, nor were the associations between drugs and resistance mutations. The EuResist database does not record which, if any, patients were left on failing regimens. Continuing a therapy in the presence of detectable viral loads is linked to increased hazard of mutation [38]. Finally, it should always be remembered that correlation does not imply causality. The underlying reason for an observed dependency could be influenced by shared inheritance from a founder virus or shared evolutionary pressures.

The current work did not investigate pathways to protease inhibitor (PI) pathways resistance as our primary interest was on cross-class RT mutational pathways. Nonetheless, some key work on pathways to protease inhibitor (PI) resistance should be noted. Many of the groups researching RTI resistance have also worked on PI resistance, and the development of statistical approaches has come from both endeavors. PI resistance mutations form clusters [19]. Temporal dependencies in HIV genetic changes in response to PIs have been indicated via Bayesian model selection [39]. Bayesian networks have been used to uncover direct influences between protein residues and treatment in clinical settings, and were able to determine the specific role of many resistance mutations against nelfinavir [40]. Phylogenetic approaches are also informative in determining pathways [13]. A cross-class pathway with promising clinical implications is a finding that patients with PI resistance are less likely to develop resistance to Bevirimat (a maturation inhibitor) than those who are PI-naive [41].

\section{Conclusions}

This investigation found specific pathways both within and across drug classes. It was further found that the most consistent factor speeding the development of NRTI resistance was not any known mutation, but rather having failed more than four previous therapies.

\section{Additional material}

Additional file 1: Supplementary materials. This file contains Figure S1, showing the relationship between event times and risk group, Table S1, showing all therapy profiles in the current data, and Table S2, the estimated hazard ratios for all identified pathways between commonly mutant locations.

\section{Acknowledgements}

Joachim Büch assisted with accessing the EuResist database. This study benefited from data provided by EuResist Network EIDB, which receives financial support via the CHAIN Project funded by the European 
Community's Seventh Framework Programme FP7/2007-2013 under grant agreement \#223131.

This work was supported by the Max Planck Institute. Support for AA was provided by the CHAIN project (EU grant HEALTH-F3-2009-223131). None of the funding organizations took part in collection, management, analysis or interpretation of the data.

\section{Author details}

Department of Computational Biology, Max Planck Institute for Informatics, Saarbrücken, Germany. ${ }^{2}$ Department of Statistical Genetics, Max Planck Institute of Psychiatry, Munich, Germany. ${ }^{3}$ Department of Biotechnology, University of Siena, Siena, Italy. ${ }^{4}$ Division of Infectious Diseases, Karolinska Institute, Stockholm, Sweden.

\section{Authors' contributions}

$G L$ designed the study, performed the statistical analysis, and wrote the text. AA assisted with database issues, interpretation of results, and insight into HIV-1 drug resistance and drug resistance testing. AT assisted with literature suggestions, modifications to the methods, and interpret the results. MZ and AS participated in gathering the data and critically revised the manuscript. $\mathrm{TL}$ provided scientific guidance, critically read the text, and suggested points of interpretation. All authors read and approved the final manuscript.

\section{Competing interests}

The authors declare that they have no competing interests.

Received: 29 March 2011 Accepted: 27 July 2011

Published: 27 July 2011

\section{References}

1. Altmann A, Beerenwinkel N, Sing T, Savenkov I, Däumer M, Kaiser R, Rhee SY, Fessel WJ, Shafer RW, Lengauer T: Improved prediction of response to antiretroviral combination therapy using the genetic barrier to drug resistance. Antivir Ther 2007, 12(2):169-178.

2. Vandamme AM, Sönnerborg A, Ait-Khaled M, Albert J, Asjo B, Bacheler L, Banhegyi D, Boucher C, Brun-Vézinet $F$, Camacho R, Clevenbergh P, Clumeck N, Dedes N, Luca AD, Doerr HW, Faudon JL, Gatti G, Gerstoft J, Hall WW, Hatzakis A, Hellmann N, Horban A, Lundgren JD, Kempf D, Miller M, Miller V, Myers TW, Nielsen C, Opravil M, Palmisano L, Perno CF, Phillips A, Pillay D, Pumarola T, Ruiz L, Salminen M, Schapiro J, Schmidt B, Schmit JC, Schuurman R, Shulse E, Soriano V, Staszewski S, Vella S, Youle M, Ziermann R, Perrin L: Updated European recommendations for the clinical use of HIV drug resistance testing. Antivir Ther 2004, 9(6):829-848.

3. Laethem $\mathrm{KV}$, Vandamme AM: Interpreting resistance data for HIV-1 therapy management-know the limitations. AIDS Rev 2006, 8:37-43.

4. Ceccherini-Silberstein F, Svicher V, Sing T, Artese A, Santoro MM, Forbici F Bertoli A, Alcaro S, Palamara G, d'Arminio Monforte A, Balzarini J, Antinori A, Lengauer T, Perno CF: Characterization and structural analysis of novel mutations in Human Immunodeficiency Virus type 1 reverse transcriptase involved in the regulation of resistance to nonnucleoside inhibitors. J Virol 2007, 81(20):11507-11519.

5. Rhee SY, Liu T, Ravela J, Gonzales MJ, Shafer RW: Distribution of Human Immunodeficiency Virus type 1 protease and reverse transcriptase mutation patterns in 4,183 persons undergoing genotypic resistance testing. Antimicrob Agents Chemother 2004, 48(8):3122-3126.

6. Cozzi-Lepri A, Ruiz L, Loveday C, Phillips AN, Clotet B, Reiss P, Ledergerber B, Holkmann C, Staszewski S, Lundgren JD, Group EIDAS: Thymidine analogue mutation profiles: factors associated with acquiring specific profiles and their impact on the virological response to therapy. Antivir Ther 2005, 10(7):791-802

7. Beerenwinkel N, Rahnenführer J, Däumer M, Hoffmann D, Kaiser R, Selbig J, Lengauer $\mathrm{T}$ : Learning multiple evolutionary pathways from crosssectional data. J Comput Biol 2005, 12(6):584-598.

8. Bacheler LT, Anton ED, Kudish P, Baker D, Bunville J, Krakowski K, Bolling L, Aujay M, Wang XV, Ellis D, Becker MF, Lasut AL, George HJ, Spalding DR, Hollis G, Abremski K: Human Immunodeficiency Virus type 1 mutations selected in patients failing efavirenz combination therapy. Antimicrob Agents Chemother 2000, 44(9):2475-2484.

9. Joly $V$, Descamps D, Peytavin G, Touati F, Mentre F, Duval X, Delarue S, Yeni $P$, Brun-Vezinet F: Evolution of Human Immunodeficiency Virus type 1 (HIV-1) resistance mutations in nonnucleoside reverse transcriptase inhibitors (NNRTIs) in HIV-1-infected patients switched to antiretroviral therapy without NNRTIs. Antimicrob Agents Chemother 2004, 48:172-175.

10. Martinez-Picado J, Martínez MA: HIV-1 reverse transcriptase inhibitor resistance mutations and fitness: a view from the clinic and ex vivo. Virus Res 2008, 134(1-2):104-123.

11. Trignetti $M$, Sing $T$, Svicher $V$, Santoro MM, Forbici F, D'arrigo $R$, Bellocchi MC, Santoro M, Marconi P, Zaccarelli M, Trotta MP, Bellagamba R, Narciso P, Antinori A, Lengauer T, Perno CF, Ceccherini-Silberstein F: Dynamics of NRTI resistance mutations during therapy interruption. AIDS Res Hum Retroviruses 2009, 2:57-64.

12. Beerenwinkel $N$, Drton M: A mutagenetic tree hidden Markov model for longitudinal clonal HIV sequence data. Biostatistics 2007, 8:53-71.

13. Buendia P, Cadwallader B, Degruttola V: A phylogenetic and Markov model approach for the reconstruction of mutational pathways of drug resistance. Bioinformatics 2009, 25(19):2522-2529.

14. Svicher $V$, Sing $T$, Santoro MM, Forbici F, Rodriguez-Barrios F, Bertoli A, Beerenwinkel N, Bellocchi MC, Gago F, d'Arminio Monforte A, Antinori A, Lengauer T, Ceccherini-Silberstein F, Perno CF: Involvement of novel Human Immunodeficiency Virus type 1 reverse transcriptase mutations in the regulation of resistance to nucleoside inhibitors. J Virol 2006, 80(14):7186-7198.

15. Arora P, Dixit NM: Timing the emergence of resistance to anti-HIV drugs with large genetic barriers. PLOS Comput Biol 2009, 5(3):e1000305.

16. Healy B, Degruttola V: Hidden Markov models for settings with intervalcensored transition times and uncertain time origin: application to HIV genetic analyses. Biostatistics 2007, 8(2):438-452.

17. Deforche K, Camacho RJ, Grossman Z, Soares MA, Laethem KV, Katzenstein DA, Harrigan PR, Kan-tor R, Shafer R, Vandamme AM, non B Workgroup: Bayesian network analyses of resistance pathways against efavirenz and nevirapine. AIDS 2008, 22(16):2107-2115.

18. Johnson VA, Brun-Vezinet F, Clotet B, Gunthard HF, Kuritzkes DR, Pillay D, Schapiro JM, Richman DD: Update of the Drug Resistance Mutations in HIV-1. Top HIV Med 2008, 16(5):138-145

19. Foulkes AS, DeGruttola V: Characterizing the Progression of Viral Mutations Over Time. Journal of the American Statistical Association 2003, 98(464):859-867.

20. JQ JF, Li R: Variable selection via nonconcave penalized likelihood and its oracle properties. JOURNAL OF THE AMERICAN STATISTICAL ASSOCIATION 2001, 96(456):1348-1360.

21. Rosen-Zvi M, Altmann A, Prosperi M, Aharoni Neuvirth $H$, Sönnerborg A, Schülter E, Struck D, Peres Y, Incardona F, Kaiser R, Zazzi M, Lengauer T: Selecting anti-HIV therapies based on a variety of genomic and clinical factors. Bioinfor-matics 2008, 24(13):i399-406[http://bioinformatics. oxfordjournals.org/cgi/content/full/24/13/i399]

22. R Development Core Team: $R$ : A language and environment for statistical computing. R Foundation for Statistical Computing, Vienna, Austria 2004 [http://www.R-project.org], [ISBN 3-900051-00-3].

23. Fan J, Feng Y, Samworth R, Wu Y: SIS: Sure Independence Screening 2010, [R package version 0.6].

24. Therneau T, original R port by Thomas Lumley: survival: Survival analysis, including penalised likelihood 2009 [http://r-forge.r-project.org], [R package version 2.35-8]

25. Wickham H: ggplot2: elegant graphics for data analysis. Springer New York 2009 [http://had.co.nz/ggplot2/book].

26. Csardi G, Nepusz T: The igraph software package for complex network research. InterJournal 2006, Complex Systems:1695[http://igraph.sf.net].

27. Brehm JH, Koontz D, Meteer JD, Pathak V, Sluis Cremer N, Mellors JW: Selection of mutations in the connection and RNase $\mathrm{H}$ domains of Human Immunodeficiency Virus type 1 reverse transcriptase that increase resistance to 3'-azido-3'-dideoxythymidine. J Virol 2007, 81(15):7852-7859.

28. Sing $T$, Svicher $V$, Beerenwinkel $N$, Ceccherini-Silberstein $F$, Däumer $M$, Kaiser R, Walter H, Korn K, Hoffmann D, Oette M, Rockstroh JK, Fätkenheuer G, Perno CF, Lengauer T: Characterization of Novel HIV Drug Resistance Mutations Using Clustering, Multidimensional Scaling and SVM-Based Feature Ranking. In Knowledge Discovery in Databases: PKDD 2005, Volume 3721 of Lecture Notes in Computer Science. Edited by: Jorge A, Torgo L, Brazdil P, Camacho R, Gama J. Springer; 2005:285-296.

29. Däumer M, Awerkiew S, Aragón SS, Kartashev Poplavskaja T, Klein R, Sichtig N, Thiele B, Lengauer T, Roomp K, Pfister H, Kaiser R: Short communication: selection of thymidine analogue resistance mutational 
patterns in children infected from a common HIV type 1 subtype $G$ source. AIDS Res Hum Retroviruses 2010, 26(3):275-278.

30. Zhang J, Hou T, Wang W, Liu JS: Detecting and understanding combinatorial mutation patterns responsible for HIV drug resistance. Proc Natl Acad Sci USA 2010, 107(4):1321-1326.

31. Koval CE, Dykes C, Wang J, Demeter LM: Relative replication fitness of efavirenz-resistant mutants of HIV-1: correlation with frequency during clinical therapy and evidence of compensation for the reduced fitness of $\mathrm{K} 103 \mathrm{~N}+\mathrm{L} 100 \mathrm{l}$ by the nucleoside resistance mutation L74V. Virology 2006, 353:184-192.

32. Weisser H, Altmann A, Sierra S, Incardona F, Struck D, Sönnerborg A, Kaiser R, Zazzi M, Tschochner M, Walter H, Lengauer T: Only slight impact of predicted replicative capacity for therapy response prediction. PLOS One 2010, 5(2):e9044.

33. Geretti AM: HIV-1 subtypes: epidemiology and significance for HIV management. Curr Opin Infect Dis 2006, 19:1-7.

34. Martínez-Cajas JL, Pant-Pai N, Klein MB, Wainberg MA: Role of genetic diversity amongst HIV-1 non-B subtypes in drug resistance: a systematic review of virologic and biochemical evidence. AIDS Rev 2008, 10(4):212-223.

35. UK Health Protection Agency: HIV and AIDS in the United Kingdom update: data to the end of March 2007. PDF 2007 [http://www.hpa.org.uk/ hpr/archives/2007/hpr1707.pdf].

36. Coutsinos D, Invernizzi CF, Xu H, Moisi D, Oliveira M, Brenner BG, Wainberg MA: Template usage is responsible for the preferential acquisition of the K65R reverse transcriptase mutation in subtype $C$ variants of human immunodeficiency virus type 1. J Virol 2009, 83(4):2029-2033.

37. Marconi VC, Sunpath H, Lu Z, Gordon M, Koranteng-Apeagyei K. Hampton J, Carpenter S, Giddy J, Ross D, Holst H, Losina E, Walker BD, Kuritzkes DR, Team SARCS: Prevalence of HIV-1 drug resistance after failure of a first highly active antiretroviral therapy regimen in KwaZulu Natal, South Africa. Clin Infect Dis 2008, 46(10):1589-1597.

38. Stürmer M, Dauer B, Moesch M, Haberl A, Mueller A, Locher L, Knecht G, Hanke N, Doerr HW, Staszewski S: Evolution of resistance mutations during lowlevel viral replication in HIV-1-infected patients treated with zidovudine/lamivudine/abacavir as a first-line regimen. Antivir Ther 2007 12:25-30.

39. Zhao JX, Foulkes AS, George El: Exploratory Bayesian model selection for serial genetics data. Biometrics 2005, 61(2):591-599.

40. Deforche K, Silander T, Camacho R, Grossman Z, Soares MA, Laethem KV, Kantor R, Moreau Y, Vandamme AM, non B Workgroup: Analysis of HIV-1 pol sequences using Bayesian Networks: implications for drug resistance. Bioinformatics 2006, 22(24):2975-2979.

41. Adamson CS, Waki K, Ablan SD, Salzwedel K, Freed EO: Impact of Human Immunodeficiency Virus type 1 resistance to protease inhibitors on evolution of resistance to the maturation inhibitor bevirimat (PA-457). Virol 2009, 83(10):4884-4894.

doi:10.1186/1742-6405-8-26

Cite this article as: Lawyer et al:: HIV-1 mutational pathways under multidrug therapy. AIDS Research and Therapy 2011 8:26.

\section{Submit your next manuscript to BioMed Central and take full advantage of:}

- Convenient online submission

- Thorough peer review

- No space constraints or color figure charges

- Immediate publication on acceptance

- Inclusion in PubMed, CAS, Scopus and Google Scholar

- Research which is freely available for redistribution

Submit your manuscript at www.biomedcentral.com/submit
Biomed Central 